\title{
Mismatch Analysis of TAB-on-Glass Connection with ACF
}

\author{
Wei-Fun Hou ${ }^{a}$, Tai-Yan Kam ${ }^{*}$ \\ Adam Hsieh $^{\mathrm{b}}$, Jian-Cheng Chen ${ }^{\mathrm{b}}$ and Shyh-Ming Chang ${ }^{\mathrm{b}}$ \\ ${ }^{a}$ Mechanical Engineering Department, National Chiao Tung University, \\ Hsin Chu, Taiwan, R. O. C. \\ ${ }^{\mathrm{b}}$ Electronics Research Laboratories, Industrial Technology Research Institute, \\ Chu Tung, Taiwan, R. O. C.
}

\begin{abstract}
Mismatch between outer lead bonds (OLBs) of a tape automated bonding (TAB) and a glass panel during fabrication is studied using the finite element method. A two-dimensional finite element model for the TAB-on Glass (TOG) connector is presented for determining the deformation of the connector. The deformation of the TOG connector induced by residual thermal stresses are determined via a two-stage approach. At the first stage, the TOG connector is under heat and pressure. The anisotropic conductive film (ACF) in-between the TAB and glass panel is assumed to be in a melting condition so that both TAB and glass panel can expand freely. At the second stage, the connector is cooled down to room temperature and the ACF becomes solidified. The deformations of the connector at the two stages are determined in the finite element analysis in which the temperature dependence of material properties is considered. Effects of particular parameters on the mismatch between the OLBs are studied by means of a number of numerical examples. A method for compensation design of the lead locations of the OLBs is proposed. It has been shown that the compensation design can produce results that will avoid the occurrence of unwanted mismatch.
\end{abstract}

\section{INTRODUCTION}

Liquid crystal display (LCD) has become an important display device in the electronic industry in recent years. In the fabrication of LCDs, Tape Automated bonding (TAB) technology by which fine pitch interconnections between LCD panels and driver-IC TAB are made possible using an anisotropic conductive film (ACF) has been adopted for packaging the components of LCDs [1-7]. When compared with the conventional packaging methods, the use of TAB package technique can provide many advantages such as having smaller and lighter packaging, reducing chip size, increasing $I / O$ pins on the chip, facilitating space reduction in LCD devices et al. Due to the advance in packaging technology, the finest interconnection pitch for LCD can be as narrow as $70 \mu \mathrm{m}$. As the interconnection pitch gets finer, the probability of having LCD failure induced by the mismatch between the outer lead bonds (OLBs) of the TAB and glass panel will be higher. The OLB mismatch is mainly caused by the thermally induced residual stresses in the cured ACF. Although some research work has been devoted to the thermal stress analysis of TOG connection, the failure of LCD induced by mismatch of OLBs is still a problem to be tackled. In this study, methods for mismatch analysis of TOG connection and compensation design of interconnection pitch are presented. The feasibility and applications of the proposed methods are demonstrated by means of a number of examples.

\section{DESCRIPTION OF THE PROBLEM}

The two-dimensional configuration of a TOG connection is shown in Fig. 1. Figs. 1a and 1b show, respectively, the connection with or without mismatch. Basically, the connection consists of three components, namely, a tape with outer leads, an ACF, and a glass panel with outer leads. The ACF is placed in-between the tape and glass panel and the three components are bonded together under heat and pressure. The curing cycle for the assembly is shown in Fig. 2. During heating, the ACF melts down and both the tape and glass panel expand freely in the lateral directions. When the assembly cools down, the ACF is solidified and the three components become an integral part. The material properties of the components are available in the literature and also listed in Table 1.

\footnotetext{
* Correspondence: Email: tykam@cc.nctu.edu.tw; Telephone: 886-3-5725634; Fax: 886-3-5728504
} 


\section{MISMATCH ANALYSIS}

The seriousness of mismatch developed in the TOG connection depends on the deformations of the three components. In the deformation analysis of the connection, the outer leads of the TAB and glass panel are not included in the formulation and their effects on the deformations of the components are neglected. Herein, the deformation analysis of the TOG connection is formulated on the basis of a two-dimensional model coupled with a two-stage deformational approach. At the first stage, the connector is under heat and pressure. The ACF is assumed to be in a melting condition and the TAB and glass panel can expand freely. The elongation of the TAB is calculated as

$$
\delta_{\mathrm{P}}=\alpha_{\mathrm{P}} \mathrm{L}_{\mathrm{P}} \Delta \mathrm{T}
$$

where $\delta, \alpha, \mathrm{L}$ denote elongation, coefficient of thermal expansion, and length, respectively; $\Delta \mathrm{T}$ is temperature rise; subscript $\mathrm{P}$ denotes TAB. The elongation of the glass panel is determined in a heat transfer analysis of the panel. At the second stage, the connector cools down to room temperature under no pressure and the ACF is transformed to a solid bar with temperature dependent material properties. The final deformations of the components of the connector are then analyzed using the two-dimensional finite element model shown in Fig. 3. It is noted that due to symmetry only one half of the TOG connector is used to establish the finite element model. The deformation analysis is then accomplished using the commercial finite element code NASTRAN [8]. Once the final deformation of the connector is determined in the above analysis, the mismatches between the outer leads of the TAB and those of the glass panel can be calculated as

$$
\mathrm{s}_{\mathrm{i}}=\widetilde{\mathrm{x}}_{\mathrm{ip}}-\widetilde{\mathrm{x}}_{\mathrm{ig}}
$$

where $s_{i}$ is the mismatch between the ith outer lead of the TAB and that of the glass panel; $\widetilde{x}_{i p}, \widetilde{x}_{i g}$ are the coordinates of the ith outer leads of the TAB and glass panel after deformation, respectively.

\section{NUMERICAL EXAMPLES}

The feasibility and applications of the aforementioned method are demonstrated by means of several examples. The material properties in Table 1 and the curing cycle of Fig. 2 are used to analyze the mismatch of the TOG connector in Fig. 1. At the first stage, the assembly is heated up from room temperature to $280^{\circ} \mathrm{C}$. In the heat transfer analysis of the assembly, it has been found that the temperature rise in the TAB is $172^{\circ} \mathrm{C}$. The temperatures at the top and bottom surfaces of the glass panel are $172^{\circ} \mathrm{C}$ and $60^{\circ} \mathrm{C}$, respectively. The elongations of the $\mathrm{TAB}$ and glass panel are $12.98 \mathrm{~mm}$ and $13.629 \mathrm{~mm}$, respectively. At the second stage, the assembly cools down to room temperature. The ACF is solidified and considered in the finite element analysis of the connector. The deformed shape of the assembly is shown in Fig. 4. The distribution of OLB mismatch across the span of the connector can be determined using Eqn. (2) and the result is shown in Fig. 5. It is noted that except for a few outer leads close to the free edge of the TAB, the OLB mismatch basically varies linearly across the span of the connector. The maximum mismatch of the connector is $13.37 \mu \mathrm{m}$ which is relatively close to the value observed in practical applications. Maximum mismatches for cases with some changes in material constants or curing parameters are also computed and the results are listed in Table 2 for comparison. It is noted that among the parameters considered, the thermal expansion coefficient of the tape may have significant effects on the OLB mismatch of the connector.

\section{COMPENSATION DESIGN OF MISMATCH}

Mismatch of OLBs will be inevitable if a uniform interconnection pitch is adopted in designing the TOG connection. Excessive OLB mismatch will be likely to cause early failure of the LCD panel. Therefore, methods for alleviating the adverse effects induced by OLB mismatch should be pursued. Herein, a nonuniform interconnection pitch approach is adopted to design the locations of the outer leads on the TAB and glass panel. The coordinates of the outer leads on the glass panel remain unchanged while those of the outer leads on the TAB are determined as

$$
\mathrm{x}_{\mathrm{iP}}^{\prime}=\mathrm{x}_{\mathrm{ig}}\left(1+\zeta_{\mathrm{g}}\right)-\mathrm{x}_{\mathrm{ip}} \zeta_{\mathrm{p}}
$$

where $\mathrm{x}_{\mathrm{ip}}, \mathrm{x}_{\mathrm{ip}}^{\prime}$ are coordinates of outer leads on tape before and after modification, respectively; $\mathrm{x}_{\mathrm{ig}}$ are the original coordinates of the outer leads on glass panel; $\zeta_{p}, \zeta_{g}$ are slopes of displacements of tape and glass panel determined at the first stage. The mismatch of the same TOG connector but with modified locations of outer leads on the TAB is also studied and the result is shown in Fig. 6. The comparison of the results in Figs. 5 and 6 shows that the adoption of the present compensation design can significantly reduce the magnitude of OLB mismatch. 


\section{CONCLUSIONS}

Mismatch between the OLBs of the TAB and glass panel for a LCD panel has been studied using a two-dimensional finite element model. Reasonable results on OLB mismatch have been obtained. The results have also revealed that the thermal expansion coefficient of TAB may have significant effects on the OLB mismatch. A compensation method for mismatch design of TOG connections has been proposed. It has been shown that the proposed compensation method can greatly reduce the magnitude of the OLB mismatch induced in the connection. It is suggested that a three-dimensional finite element model be used in the mismatch analysis if a more accurate prediction of OLB mismatch is desired.

\section{REFERENCES}

1. H. Atarashi, N. Kakimoto, H. Matsubrara, K. Yamamura, T. Mukii, and H. Matsui, "Chip-on-Glass technology using conductive particles and light-setting adhesives," Proceedings of 1990 Japan International Electronic Manufacturing Technology Symposium, June 9-11, 1990, Tokyo, Japan, pp. 190-195.

2. H. Matsubara, H. Atarashi, K. Yamamura, N. Kakimoto, K. Naitoh, and T. Nukii, "Bare-chip face-down bonding technology using conductive particles and lightsetting adhesives:ELASTIC method," IMC 1992 Proceeding, Yokohama, pp. 81-87, 1992.

3. H. Yoshida, et al, "Packaging method of liquid crystal driver LSIs by Tape-On-Panel technology," ISHM '88 proceeding, pp. 307-313.

4. F. Juskey, "Heat seal connector: a new high density SMT interconnection system," IEEE/CHMT '89 IEMT symposium, pp. 121-125.

5. J. C. Hwang, "Advanced Low-Cost Bare-Die Packaging Technology for Liquid Crystal Displays," IEEE/CPMT Int'1 Electronics Manufacturing Technology Symposium, 1994, pp. 199-202.

6. N. R. Basavanhally, D. D. Chang, B. H. Cranston, "Direct Chip Interconnect with Adhesive-Connector Films," Proceeding of Electronic of Electronic Components and Technology Conference, 1992, pp.498-491.

7. R. Ascenbrenner, A. Ostrmann, G. Motulla, E. Zakel, H. Reichl, "Flip Chip Attachment Using Anisotropic Conductive Adhesives and Electronic Nickel Bumps," IEEE Transactions on Components, Packaging and Manufacturing Technology - Part C, Vol. 20, No. 2, April 1997, pp.95-100.

8. “MSC/NASTRAN Version 66," MacNeal-Schwendler Corp., Los Angels, CA, Dec 1990. 
Table 1. Material constants for components of TOG connection

\begin{tabular}{|c|c|c|c|c|c|}
\hline $\begin{array}{l}\text { Component } \\
\text { Constant }\end{array}$ & \multicolumn{2}{|c|}{ Tape } & \multicolumn{2}{|c|}{$\mathrm{ACF}$} & Glass \\
\hline \multirow{4}{*}{$\mathrm{E}(\mathrm{MPa})$} & \multirow{4}{*}{\multicolumn{2}{|c|}{8826}} & $0^{\circ} \mathrm{C}$ & 2548 & \multirow{4}{*}{70000} \\
\hline & & & $40^{\circ} \mathrm{C}$ & 2548 & \\
\hline & & & $121^{\circ} \mathrm{C}$ & 1500 & \\
\hline & & & $200^{\circ} \mathrm{C}$ & 1500 & \\
\hline$v$ & \multicolumn{2}{|c|}{0.3} & \multicolumn{2}{|c|}{0.3} & 0.2 \\
\hline \multirow{5}{*}{$\alpha\left(\times 10^{-6} /{ }^{\circ} \mathrm{C}\right)$} & $0^{\circ} \mathrm{C}$ & 8 & $0^{\circ} \mathrm{C}$ & 52.2 & \multirow{5}{*}{4.7} \\
\hline & $99^{\circ} \mathrm{C}$ & 8 & $110^{\circ} \mathrm{C}$ & 52.2 & \\
\hline & $100^{\circ} \mathrm{C}$ & 9 & $111^{\circ} \mathrm{C}$ & 177.6 & \\
\hline & $101^{\circ} \mathrm{C}$ & 10 & $112^{\circ} \mathrm{C}$ & 303 & \\
\hline & $300^{\circ} \mathrm{C}$ & 10 & $300^{\circ} \mathrm{C}$ & 303 & \\
\hline
\end{tabular}

Table 2. Maximum mismatches for TOG connectors with different parameters

\begin{tabular}{|c|c|}
\hline Modified parameter & Maximum mismatch $(\mu \mathrm{m})$ \\
\hline$\alpha_{\mathrm{G}}=3.8 \times 10^{-6} /{ }^{\circ} \mathrm{C}$ & 14.43 \\
\hline$\alpha_{\mathrm{P}}=5 \times 10^{-6} /{ }^{\circ} \mathrm{C}$ & 5.90 \\
\hline $\mathrm{T}_{\mathrm{P}}=165^{\circ} \mathrm{C}$ & 12.65 \\
\hline
\end{tabular}



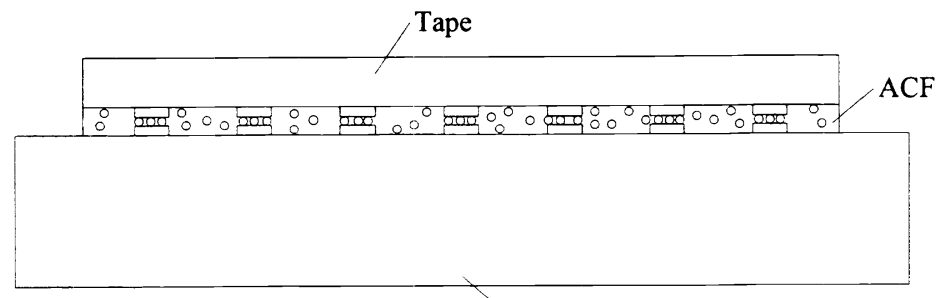

glass panel

(a)

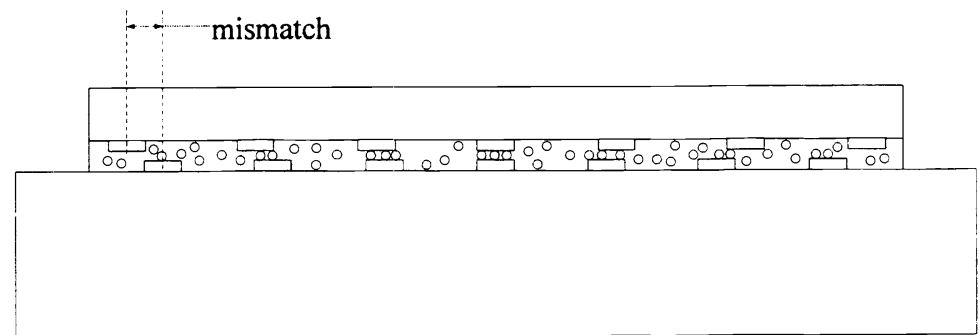

(b)

Fig. 1. 2-D view of TOG connection

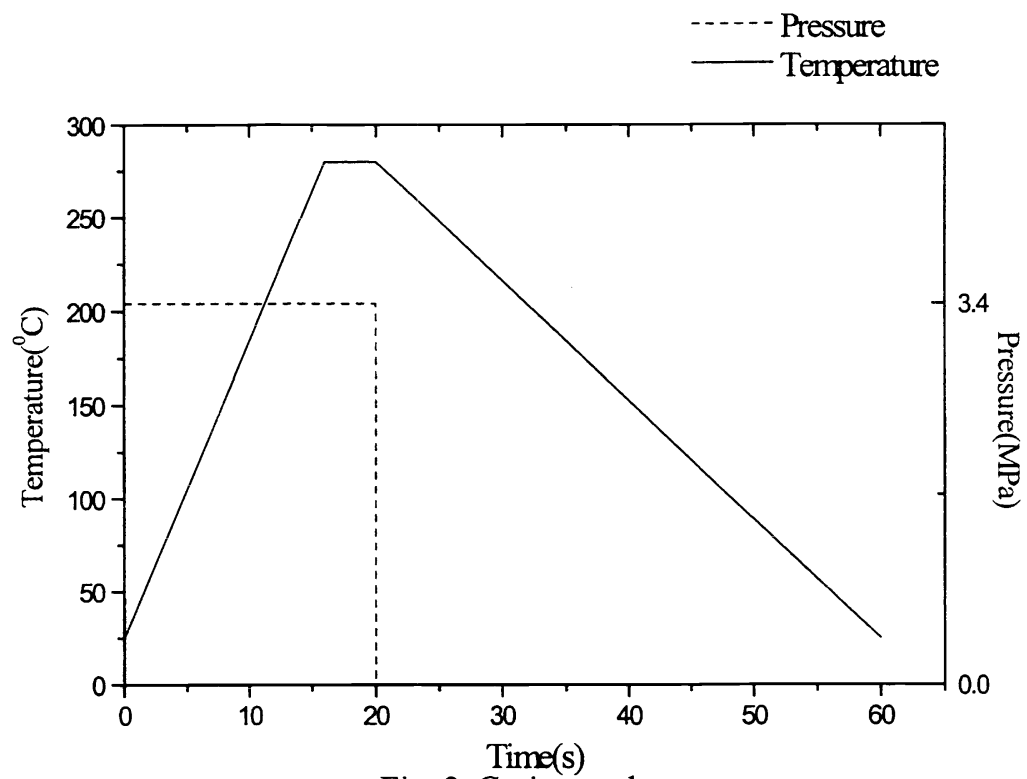

Fig. 2. Curing cycle 


\section{Tape}

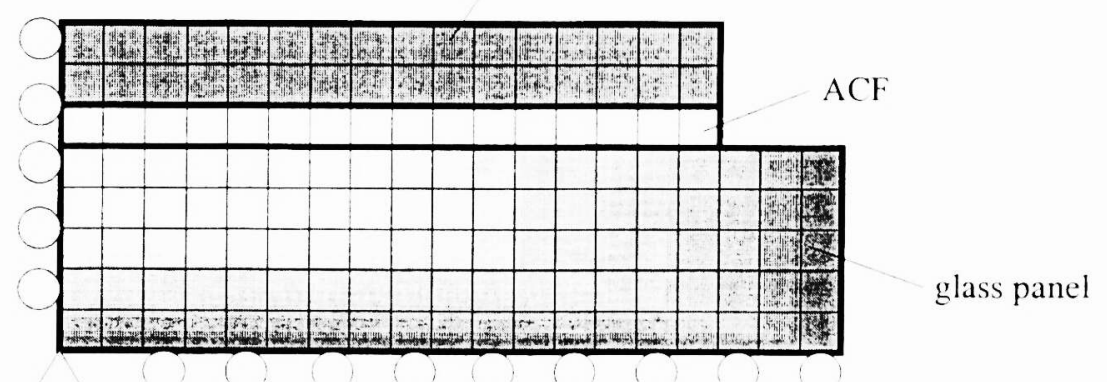

Fig. 3 Finite element model of connector

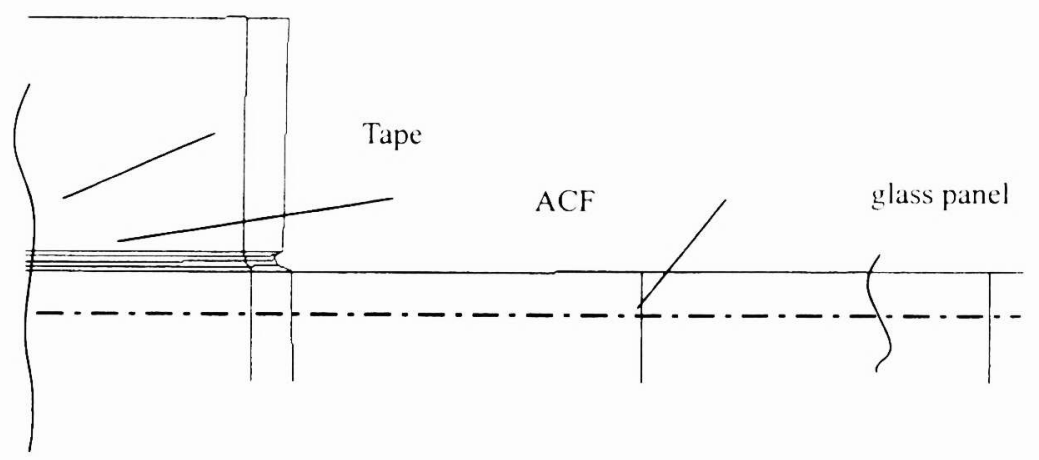

Fig. 4. Deformed shape of TOG connector 


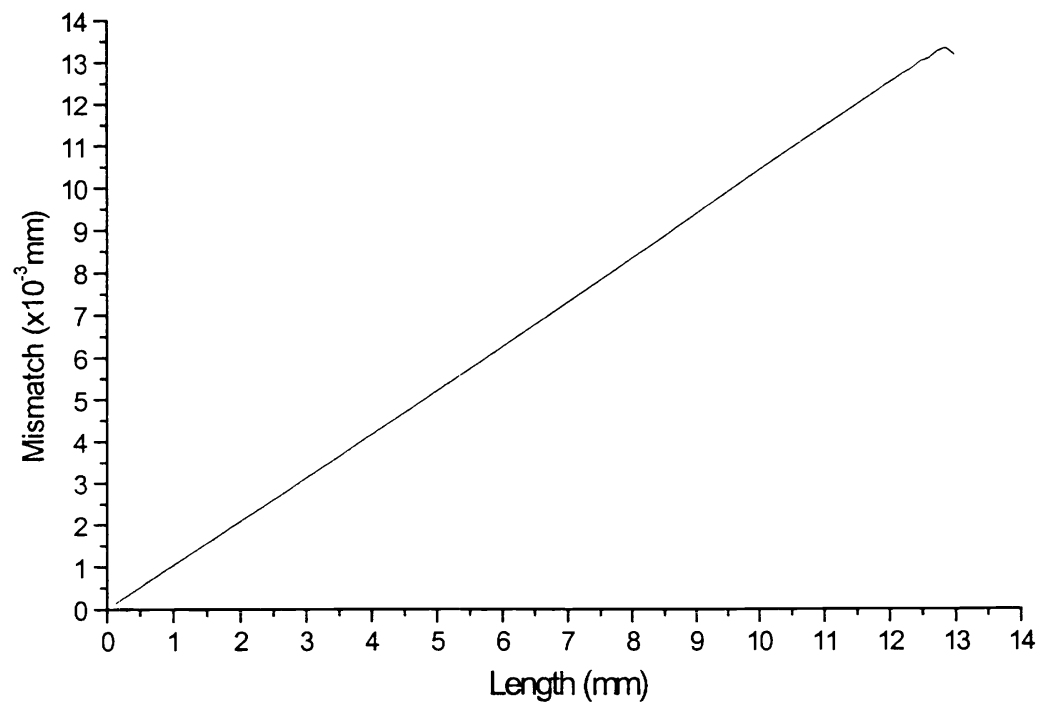

Fig. 5. Distribution of mismatch

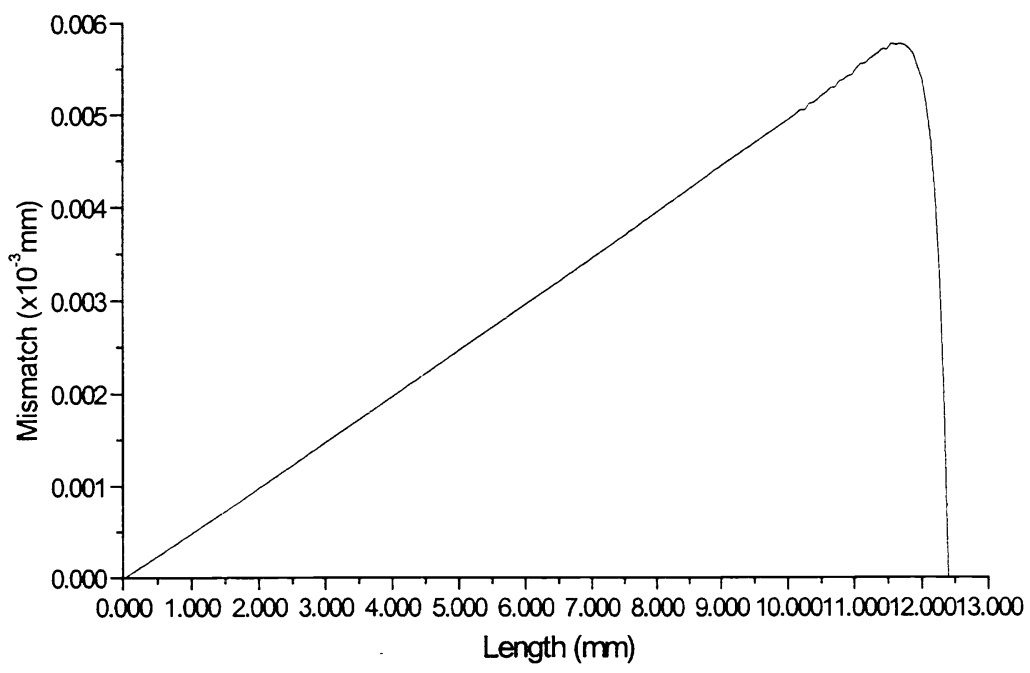

Fig. 6. Mismatch of TOG connection with compensation design 